\title{
Association analysis of vitamin D receptor gene polymorphisms and bone mineral density in postmenopausal Mexican-Mestizo women
}

\author{
A. González-Mercado ${ }^{1,2}$, J.Y. Sánchez-López ${ }^{1,2}$, J.A. Regla-Nava ${ }^{1, a}$, \\ J.I. Gámez-Nava ${ }^{3,4}$, L. González-López ${ }^{5,6}$, J. Durán-González ${ }^{1,2}$, \\ A. Celis ${ }^{3,6}$, F.J. Perea-Díaz ${ }^{1,2}$, M. Salazar-Páramo ${ }^{4,7}$ and B. Ibarra ${ }^{1,2}$ \\ ${ }^{1}$ División de Genética, Centro de Investigación Biomédica de Occidente, \\ IMSS, Guadalajara, Jalisco, México \\ ${ }^{2}$ Doctorado en Genética Humana, Universidad de Guadalajara, Guadalajara, \\ Jalisco, México \\ ${ }^{3}$ Unidad de Investigación Médica en Epidemiología Clínica, \\ Hospital de Especialidades, CMNO, IMSS, Guadalajara, Jalisco, México \\ ${ }^{4}$ Doctorado en Farmacología, Departamento de Fisiología, CUCS, \\ Universidad de Guadalajara, Guadalajara, Jalisco, México \\ ${ }^{5}$ Servicio de Reumatología del HGR110 IMSS, Guadalajara, Jalisco, México \\ ${ }^{6}$ Doctorado en Salud Pública, Departamento de Salud Pública, CUCS, \\ Universidad de Guadalajara, Guadalajara, Jalisco, México \\ ${ }^{7}$ División de Investigación en Salud, Hospital de Especialidades, CMNO, \\ IMSS, Guadalajara, Jalisco, México
}

Genet. Mol. Res. 12 (3): 2755-2763 (2013)

Received January 21, 2013

Accepted May 24, 2013

Published July 30, 2013

DOI http://dx.doi.org/10.4238/2013.July.30.13

aPresent address: Department of Molecular and Cell Biology, Centro Nacional de Biotecnología (CNB-CSIC), Madrid, Spain Corresponding author: B. Ibarra

E-mail: bibarra@mail.udg.mx

\begin{abstract}
We investigated associations between vitamin D receptor $(V D R)$ gene polymorphisms, FokI T $>\mathrm{C}$ (rs2228570), BsmI $\mathrm{G}>\mathrm{A}$ (rs1544410), ApaI G $>\mathrm{T}$ (rs7975232), and TaqI T $>\mathrm{C}$ (rs731236), with bone mineral density (BMD) in postmenopausal Mexican-Mestizo women. Three hundred and twenty postmenopausal women participated,
\end{abstract}


who were classified according to World Health Organization criteria as non-osteoporotic (Non-OP; $\mathrm{N}=88$ ), osteopenic (Opn; $\mathrm{N}=144$ ), and osteoporotic (OP; $\mathrm{N}=88$ ). BMD measurements at the lumbar (L1-L4) spine and at the left and right femoral neck were obtained by dual-energy X-ray absorptiometry. Single nucleotide polymorphisms (SNPs) were genotyped using real-time polymerase chain reaction and TaqMan probes. Genotype and allelic frequencies of the 4 VDR SNPs were similar among the 3 groups. Polymorphic allele frequencies were as follows: FokI (C) 0.53, 0.49, 0.56; BsmI (A) 0.26, 0.22, 0.23; ApaI (T) 0.43, 0.39, 0.44; TaqI (C) 0.27, 0.22, 0.23 for the Non-OP, Opn, and $\mathrm{OP}$ groups, respectively. Although no associations were found between the SNPs and BMD, based on the putative function of the FokI SNP, we constructed, for the first time, the haplotype with the 4 VDR SNPs, and found that the CGGT haplotype differed between the Non$\mathrm{OP}$ and $\mathrm{OP}$ groups $(21.8$ vs $31.8 \%, \mathrm{P}<0.05)$. The risk analysis for this haplotype was nearly significant under the dominant model $(\mathrm{OR}=$ $1.783,95 \% \mathrm{CI}=0.98-3.25, \mathrm{P}=0.058)$. This result suggests a possible susceptibility effect of the C allele of the FokI SNP for the development of osteoporosis in postmenopausal Mexican-Mestizo women.

Key words: Bone mineral density; Postmenopausal women; Osteoporosis; VDR polymorphisms

\section{INTRODUCTION}

Osteoporosis (OP) is a complex disease mediated by the interaction of environmental factors and genes that can individually affect bone mineral density (BMD). This disease is a public health problem that affects postmenopausal women and predisposes them to an increased risk of osteoporotic fractures. OP is associated with considerable morbidity and mortality, particularly in older women (Anonymous, 2010). It is estimated that $46-62 \%$ of BMD variation can be attributed to genetic factors, including variations in expressions of different candidate genes encoding proteins such as calciotropic hormones, cytokines, growth factors, bone matrix proteins, and growth factor receptors, which all participate in bone metabolism and in the pathogenesis of OP (Gronholz, 2008; Marini and Brandi, 2010). The vitamin D receptor $(V D R)$ gene has been extensively studied in relation to BMD, with inconsistent results reported for different populations around the world (Thakkinstian et al., 2004; Zintzaras et al., 2006; Cashman and Seamans, 2007; Marini and Brandi, 2010). The VDR gene, also known as the 1,25-dihydroxy vitamin D3 receptor, is located on chromosome 12q13.11, is $75 \mathrm{~kb}$ in size, and is composed of 11 exons. Exons 2 and 3 encode the amino acids involved in DNA binding, and exons 7, 8, and 9 are implicated in vitamin D binding. The VDR protein is a member of the superfamily of nuclear receptors that function as transcription factors (OMIM, 2012). The VDR gene contains many single nucleotide polymorphisms (SNPs), although FokI T $>\mathrm{C}$ (rs2228570), BsmI G>A (rs1544410), ApaI G>T (rs7975232), and TaqI T $>\mathrm{C}$ (rs731236) are the most widely SNPs studied of this gene (Zintzaras et al., 2006). The FokI polymorphism is a $\mathrm{T} / \mathrm{C}$ transition at the translation initiation site of exon 2 in the $5^{\prime}$ coding region of the VDR gene. This change creates a new start codon (ATG to $\mathrm{A} \underline{\underline{C}}$ ) resulting in a peptide that is shorter 
by 3 amino acids ( 427 compared with 424), which has higher transcriptional activity compared with the full-length vitamin D receptor protein (Alimirah et al., 2011; OMIM, 2012).

The prevalence of OP in postmenopausal Mexican women varies between 8.3 and $19.8 \%$, depending on geographic region (Murrillo-Uribe et al., 1999; Guzmán Ibarra et al., 2003; Mendoza-Romo et al., 2003; Sen et al., 2005; González-Arellano et al., 2007), and we recently found a prevalence of $25 \%$ in Western Mexico (González-Mercado A, SalazarPáramo M, Celis A, Gámez-Nava JI, et al., unpublished results). In the general Mexican population, the OP prevalence is between 9.7 and 17.9\% (De Lago Acosta et al., 2008; Tamayo et al., 2009). Two studies related to $V D R$ and OP in postmenopausal women in Mexico has been conducted to date. Jaramillo-Rangel et al. (1999) investigated the association using the BsmI, ApaI, and TaqI polymorphisms in 98 Mexican women over 25 years of age from Mexico City (79 postmenopausal), and did not find any association of these SNPs with BMD. The second study, conducted by Lisker et al. (2003), focused on the BsmI and FokI polymorphisms in postmenopausal Mexican women ( 65 with OP and 57 without OP), and found higher frequencies of the bb (GG) genotype of BsmI (the wild-type allele) in the OP group, suggesting a susceptibility role of allele $b$ for OP.

The aim of the present study was to analyze the associations between the FokI T $>C$, $B s m \mathrm{I} G>\mathrm{A}, A p a \mathrm{I} \mathrm{G}>\mathrm{T}$, and TaqI T>C polymorphisms and haplotypes of the $V D R$ gene with BMD in Mexican-Mestizo postmenopausal women from Western Mexico.

\section{MATERIAL AND METHODS}

\section{Subjects}

Three hundred and twenty postmenopausal Mexican-Mestizo women from Western Mexico were included in the study, which were recruited from the Hospital General Regional No. 110 of the Instituto Mexicano del Seguro Social in Guadalajara city. They were classified into 3 groups, according to World Health Organization criteria, based on BMD measurements at the lumbar (L1-L4) spine: 88 non-osteoporotic women (Non-OP; t score > -1 SD), 144 osteopenic women (Opn; $\mathrm{t}$ score between -2.5 and $-1 \mathrm{SD}$ ), and 88 OP women (t score $<-2.5$ $\mathrm{SD})$. The groups were similar in age and body mass index (BMI) $(\mathrm{P}>0.05$; Table 1). BMD measurements at the lumbar (L1-L4) spine and at the left and right femoral neck were obtained by an individual trained in dual-energy X-ray absorptiometry (Prodigy Advance, GE) following manufacturer protocols. A structured questionnaire assessing biological and lifestyle risk factors was completed by each subject, who all signed an informed consent form. The protocol was approved by a local Ethics Committee.

\section{Genotyping}

Genomic DNA was isolated from peripheral blood leukocytes and DNA extraction was performed using the salting out precipitation method (Miller et al., 1988). The VDR SNPs, FokI $\mathrm{T}>\mathrm{C}$ (rs2228570), BsmI G>A (rs1544410), ApaI G>T (rs7975232), and TaqI T>C (rs731236), were genotyped using a TaqMan 5'-exonuclease SNP allelic discrimination assay with the ABI 7300 Real-Time PCR System (Applied Biosystems, Foster City, CA, USA). Negative and positive controls were included to ensure accuracy of genotyping. The reactions were carried out in a total volume of $15 \mu \mathrm{L}$ containing $50 \mathrm{ng}$ DNA. The thermal cycler conditions consisted of an initial hold for $10 \mathrm{~min}$ at $95^{\circ} \mathrm{C}$, followed by 40 cycles of $15 \mathrm{~s}$ at $92^{\circ} \mathrm{C}$, and $1 \mathrm{~min}$ at $60^{\circ} \mathrm{C}$ each. 


\section{Statistical analyses}

Differences between the Non-OP, Opn, and OP groups in age, BMI, menopause or menarche status, BMD (lumbar spine L1-L4 and left and right femoral neck), personal history of fractures, presence of pathologic fractures, family history of OP and fractures, alcoholism, smoking, coffee intake, and sedentarism of subjects were analyzed by the Student $t$-test and the chi-square test using SPSS version 16.0 (SPSS Inc., Chicago, IL, USA). P values $<0.05$ were considered to be statistically significant.

The frequency distributions of $V D R$ genotypes and alleles in Non-OP, Opn, and OP postmenopausal women were compared for each SNP studied using the chi-square test. Hardy-Weinberg equilibrium (HWE) was tested in each SNP and group of participants using the chi-square test.

The relationship of $V D R$ genotypes with age, weight, height, BMI, menopause, menarche, and BMD (lumbar spine L1-L4 and left and right femoral neck) variables were tested by analysis of variance (ANOVA) in the Non-OP, Opn, and OP groups. An odds ratio (OR) analysis was performed using the Cochran Armitage test under classical inheritance patterns, considering the polymorphic alleles FokI C, BsmI A, ApaI T, and TaqI C as risk alleles between Non-OP and OP groups. The D' values of linkage disequilibrium (LD) and the haplotypes of the VDR SNPs were assessed using the Arlequin program version 3.01. An ANOVA was also performed to investigate the relationship between the haplotypes and the quantitative variables.

\section{RESULTS}

The age, BMI, and BMD of the Non-OP, Opn, and OP postmenopausal MexicanMestizo women included in the study are shown in Table 1. It is noteworthy that the BMI of the 3 groups are considered to be overweight according to the World Health Organization classification. As expected, the OP group showed the lowest BMD values, and BMD values were higher in the lumbar spine than in the femoral neck in all 3 groups (Table 1). No statistically significant differences were observed among the 3 groups with respect to menopause, menarche, personal history of fractures, presence of pathologic fractures, family history of OP and fractures, alcoholism, smoking, coffee intake, or sedentarism.

$\begin{aligned} & \text { Table 1. Age, body mass index (BMI) and bone mineral density (BMD) of the postmenopausal Mexican-Mestizo } \\
& \text { women studied. }\end{aligned}$
\begin{tabular}{lccc} 
& \\
\hline & Non-osteoporotic & Osteopenic & Osteoporotic \\
\hline Number & 88 & 144 & 88 \\
Age $($ years)* & $56.34 \pm 4.98$ & $57.53 \pm 5.92$ & $57.65 \pm 5.58$ \\
BMI $\left(\mathrm{kg} / \mathrm{m}^{2}\right)^{*}$ & $29.33 \pm 4.30$ & $29.34 \pm 4.18$ & $28.48 \pm 4.03$ \\
Lumbar spine L1-L4 BMD $\left(\mathrm{g} / \mathrm{cm}^{2}\right)^{* *}$ & $1.174 \pm 0.108$ & $0.976 \pm 0.047$ & $0.833 \pm 0.054$ \\
Femoral neck left BMD $\left(\mathrm{g} / \mathrm{cm}^{2}\right)^{* *}$ & $1.004 \pm 0.087$ & $0.876 \pm 0.097$ & $0.789 \pm 0.086$ \\
Femoral neck right BMD $\left(\mathrm{g} / \mathrm{cm}^{2}\right)^{* *}$ & $1.002 \pm 0.083$ & $0.886 \pm 0.102$ & $0.788 \pm 0.086$ \\
\hline
\end{tabular}

$* \mathrm{P}=\mathrm{NS}, * * \mathrm{P}<0.05$, between the three groups (Student $t$-test). Values are reported as means \pm standard deviation.

No statistically significant differences were revealed in the genotype and allele frequencies of the 4 SNPs of the $V D R$ gene among the Non-OP, Opn, and OP postmenopausal women groups (Table 2). Distributions of genotype frequencies for all 4 SNPs were in HWE. 


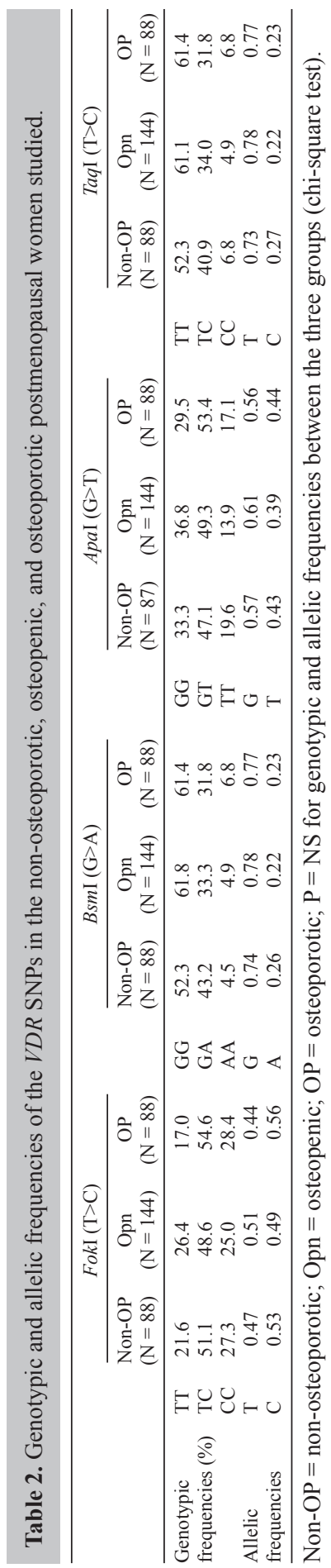


ANOVA revealed that the genotypes of the 4 SNPs studied were not associated with age, BMI, menopause, and BMD in the 3 groups $(\mathrm{P}>0.05)$. We only found statistically significant differences for ApaI in the Opn group between the GG and GT genotypes for menarche (GG 12.51 \pm 2.28 ; GT $13.45 \pm 1.65$ years, $\mathrm{P}=0.024$ ); however, this observation needs further investigation.

The association analysis for all $V D R$ alleles was not found to be significant using any of the classical inheritance models (dominant, recessive, codominant, or additive). We found evidence of strong LD ( $\mathrm{D}^{\prime}>0.5$ ) within Bsm I-ApaI, BsmI-TaqI, and ApaI-TaqI in the 3 groups, which is in agreement with results of Fang et al. (2005).

Considering the 3 VDR SNPs in LD, we observed 8 possible haplotypes, with GGT and ATC being the most common among the 3 groups with similar frequency distributions (Table 3). In particular, the ATC haplotype showed statistically significant distribution between the Non-OP and Opn groups $(\mathrm{P}<0.05)$, as did the AGC haplotype, since it was only observed in the Opn group $(\mathrm{P}<0.05)$ (Table 3$)$.

Table 3. Haplotype distributions of the VDR single nucleotide polymorphisms (SNPs) in the non-osteoporotic, osteopenic, and osteoporotic postmenopausal women studied.

\begin{tabular}{|c|c|c|c|c|c|c|c|}
\hline $\begin{array}{l}\text { Haplotype } \\
(\text { Bsm I, ApaI, and TaqI) }\end{array}$ & $\begin{array}{c}\text { Non-OP } \\
N_{\mathrm{A}}=174 \\
\quad(\%)\end{array}$ & $\begin{array}{c}\text { Opn } \\
N_{\mathrm{A}}=288 \\
(\%)\end{array}$ & $\begin{array}{c}\text { OP } \\
N_{\mathrm{A}}=176 \\
(\%)\end{array}$ & $\begin{array}{l}\text { Haplotype } \\
(\text { FokI, BsmI, ApaI, and TaqI) }\end{array}$ & $\begin{array}{c}\text { Non-OP } \\
N_{\mathrm{A}}=174 \\
(\%)\end{array}$ & $\begin{array}{c}\text { Opn } \\
N_{\mathrm{A}}=288 \\
(\%)\end{array}$ & $\begin{array}{c}\mathrm{OP} \\
N_{\mathrm{A}}=176 \\
(\%)\end{array}$ \\
\hline G G T & 52.9 & 61.5 & 54.5 & T G G T & 31.0 & $34.7 *$ & $22.9 *$ \\
\hline A T C & $24.2 *$ & $16.0 *$ & 20.5 & $\mathrm{CGGT}$ & $21.8 *$ & 26.7 & $31.8^{*}$ \\
\hline G T T & 17.3 & 16.0 & 20.5 & C A T C & $13.8 *$ & $5.9^{*}$ & 7.9 \\
\hline $\mathrm{G} \mathrm{G} \mathrm{C}$ & 2.3 & 0.0 & 1.1 & $\mathrm{C} \mathrm{G} \mathrm{T} \mathrm{T}$ & 13.2 & 9.7 & 12.5 \\
\hline $\mathrm{ATT}$ & 1.1 & 0.0 & 1.7 & T A T C & 10.3 & 10.1 & 12.5 \\
\hline $\mathrm{AGT}$ & 1.1 & 0.3 & 0.6 & T G T T & 4.0 & 6.3 & 7.9 \\
\hline $\mathrm{G} \mathrm{T} \mathrm{C}$ & 1.1 & 0.6 & 1.1 & $\mathrm{CG} \mathrm{G} \mathrm{C}$ & 2.3 & 0.0 & 1.1 \\
\hline \multirow[t]{6}{*}{$\mathrm{AGC}$} & $0.0^{*}$ & $5.6^{*}$ & $0.0^{*}$ & T G T C & 1.2 & 0.0 & 1.1 \\
\hline & & & & $\mathrm{CATT}$ & 1.2 & 0.0 & 1.7 \\
\hline & & & & $\mathrm{CAGT}$ & 0.6 & 0.3 & 0.0 \\
\hline & & & & T A G T & 0.6 & 0.0 & 0.6 \\
\hline & & & & $\mathrm{C} \mathrm{G} \mathrm{T} \mathrm{C}$ & 0.0 & 0.7 & 0.0 \\
\hline & & & & $\mathrm{CAGC}$ & $0.0^{*}$ & $5.6^{*}$ & $0.0^{*}$ \\
\hline
\end{tabular}

$N_{\mathrm{A}}=$ number of alleles. Non-OP $=$ non-osteoporotic; Opn = osteopenic; $\mathrm{OP}=$ osteoporotic; $* \mathrm{P}<0.05$ (chi-square test); $\mathrm{ATC}=$ Non-OP compared with Opn; AGC $=$ Opn compared with Non-OP and OP; TGGT = Opn compared with OP; CGGT $=$ Non-OP compared with OP; CATC $=$ Non-OP compared with Opn; CAGC $=$ Opn compared with Non-OP and OP.

On the other hand, we found that the FokI SNP was not in LD with BsmI, ApaI, and TaqI, which is in agreement with results of previous studies (Fang et al., 2005). However, considering the functional role of the FokI polymorphism, we also included this site in the construction of the haplotypes, and observed that 13 of the 16 possible haplotypes showed no statistically significant differences among the 3 groups using a pairwise distribution test (Table 3 ), with the TGGT and CGGT haplotypes being the most common. The frequency of the TGGT haplotype differed significantly between the Opn and the OP groups (34.7vs 22.9\%, P $<0.05)$ and the CGGT haplotype also differed significantly between the Non-OP and the OP groups $(21.8$ vs $31.8 \%, \mathrm{P}<0.05)$. In addition, the frequency of the CATC haplotype differed significantly between the Non-OP and the Opn groups (13.8 vs 5.9\%, P <0.05), and the CAGC haplotype differed between the Opn group and the Non-OP and OP groups, as it was only observed in the Opn group (5.6\%) (Table 3). 
The OR analysis of the haplotypes did not reveal any statistically significant association with OP. However, we observed a marginally significant $\mathrm{P}$ value for the CGGT haplotype using the dominant model $(\mathrm{OR}=1.783,95 \% \mathrm{CI}=0.98-3.25, \mathrm{P}=0.058)$. Considering CGGT as the risk haplotype, we performed ANOVA, including all variables described in the Material and Methods section, in the 3 groups individually as well as in the total sample $(\mathrm{N}=320)$ and found no significant results.

\section{DISCUSSION}

OP is a multifactorial disease with a large genetic component that represents a worldwide public health problem. VDR polymorphisms have been among the most widely SNPs studied in OP. In particular, Bsm I has shown inconsistent results with respect to its association with BMD (Thakkinstian et al., 2004; Zintzaras et al., 2006; Li et al., 2012; Jia et al., 2013; Qin et al., 2013).

We analyzed the FokI, BsmI, ApaI, and TaqI polymorphisms of the VDR gene in postmenopausal Mexican-Mestizo women, and results showed that genotypic and allelic frequencies were similar between the Non-OP, Opn, and OP groups. This suggests that there is no association between these VDR SNPs with OP in this Mexican population. This lack of association was also observed in a recent meta-analysis (Qin et al., 2013).

In regard to other studies performed in Mexican populations, allelic frequencies of BsmI, ApaI, and TaqI SNPs in postmenopausal women of the present study were similar to those observed by Jaramillo-Rangel et al. (1999) in postmenopausal women from the central region of Mexico. However, we cannot completely discard an association of VDR SNPs with BMD in Mexican postmenopausal women from another region of the country, since several studies have demonstrated ethnic and genetic diversity in distinct regions of Mexico (RubiCastellanos et al., 2009; Silva-Zolezzi et al., 2009). On the other hand, comparing our results with those reported by Lisker et al. (2003) reveals statistically significant differences in the frequencies of the A allele for $B s m \mathrm{I}$ in the Non-OP group ( 0.261 compared to 0.561 , respectively, $\mathrm{P}<0.0001)$ and in the OP group $(0.227$ compared to 0.358 , respectively, $\mathrm{P}=0.013)$; a protective role against OP was proposed for this allele by Lisker et al. (2003). Results of Jaramillo-Rangel et al. (1999), Lisker et al. (2003), and the present study in Mexican populations suggest that the role of a specific allele can be different depending on genetic background and lifestyle, as has been observed in other multifactorial diseases.

The relationship between genotype and BMD was similar across the 3 skeletal sites (lumbar spine, left and right femoral neck) in the Non-OP, Opn, and OP groups; however, in the OP group, a discrete difference in the BMD of the lumbar spine between the TT and TC FokI genotypes could be observed (TT: $0.863 \pm 0.048$, TC: $0.825 \pm 0.048$; ANOVA, P $=0.057$, Bonferroni's correction, $\mathrm{P}=0.057$ ), which suggested that statistically significant differences might be observed with a larger sample size.

The OR analysis with the 3 linked SNPs, BsmI-ApaI-TaqI haplotype, was not associated with OP; however, for the first time, we constructed complete haplotypes that included all of the alleles, FokI T>C, BsmI G $>$ A, ApaI G $>$ T, and TaqI T $>$ C, because the VDR protein from the polymorphic $\mathrm{C}$ allele of FokI has higher transcriptional activity on target genes in response to vitamin $\mathrm{D}$ when this shorter peptide is compared with the full-length VDR isoform (Alimirah et al., 2011).

We found that only the CGGT haplotype differed between the Non-OP and OP groups 
(21.8 vs 31.8\%, $\mathrm{P}<0.05)$, and in the risk analysis, we observed an OR of 1.783 for this haplotype $(95 \% \mathrm{CI}=0.98-3.25, \mathrm{P}=0.058)$ using the dominant model. As these data are nearly significant, this may suggest a possible susceptibility effect that could be attributable to the $\mathrm{C}$ allele of the FokI SNP. We believe that the effect of the CGGT haplotype on the susceptibility of the development of osteoporosis in postmenopausal Mexican-Mestizo women could be better clarified with a larger sample.

\section{ACKNOWLEDGMENTS}

Research supported by a research grant from Consejo Nacional de Ciencia y Tecnología (\#2003-107). The authors acknowledge Erika A. Aguilar-Chávez, Juan J. Ruelas-Medina, and Joaquín Rojo-Contreras for their valuable help with data collection.

\section{REFERENCES}

Anonymous (2010). Management of osteoporosis in postmenopausal women: 2010 position statement of The North American Menopause Society. Menopause 17: 25-54.

Alimirah F, Peng X, Murillo G and Mehta RG (2011). Functional significance of vitamin D receptor FokI polymorphism in human breast cancer cells. PLoS One 6: e16024.

Cashman KD and Seamans K (2007). Bone health, genetics, and personalised nutrition. Genes Nutr. 2: 47-51.

De Lago Acosta A, Parada Tapia MG and Somera Iturbide J (2008). Osteoporosis prevalence in open population at Mexico City. Ginecol. Obstet. Mex. 76: 261-266.

Fang Y, van Meurs JB, d'Alesio A, Jhamai M, et al. (2005). Promoter and 3'-untranslated-region haplotypes in the vitamin $\mathrm{d}$ receptor gene predispose to osteoporotic fracture: the rotterdam study. Am. J. Hum. Genet. 77: 807-823.

González-Arellano JA, Milla-Villeda RH, Hernández-Vera GE, Cisneros-Pérez V, et al. (2007). Prevalence of osteoporosis and osteopenia among women over fifty years of age, from the city of Durango, Mexico, diagnosed by forearmDEXA. Gac. Med. Mex. 143: 365-369.

Gronholz MJ (2008). Prevention, diagnosis, and management of osteoporosis-related fracture: a multifactoral osteopathic approach. J. Am. Osteopath. Assoc. 108: 575-585.

Guzmán Ibarra M, Ablanedo Aguirre J, Armijo Delgadillo R and García Ruíz Esparza M (2003). Prevalence of osteopenia and osteoporosis assessed by densitometry in postmenopausal women. Ginecol. Obstet. Mex. 71: 225-232.

Jaramillo-Rangel G, Cerda-Flores RM, Cardenas-Ibarra L, Tamayo-Orozco J, et al. (1999). Vitamin D receptor polymorphisms and bone mineral density in Mexican women without osteoporosis. Am. J. Hum. Biol. 11: 793-797.

Jia F, Sun RF, Li QH, Wang DX, et al. (2013). Vitamin D receptor BsmI polymorphism and osteoporosis risk: a metaanalysis from 26 studies. Genet. Test. Mol. Biomarkers 17: 30-34.

Li Y, Xi B, Li K and Wang C (2012). Association between vitamin D receptor gene polymorphisms and bone mineral density in Chinese women. Mol. Biol. Rep. 39: 5709-5717.

Lisker R, López MA, Jasqui S, Ponce De León Rosales S, et al. (2003). Association of vitamin D receptor polymorphisms with osteoporosis in mexican postmenopausal women. Hum. Biol. 75: 399-403.

Marini F and Brandi ML (2010). Genetic determinants of osteoporosis: common bases to cardiovascular diseases? Int. J. Hypertens. 2010.

Mendoza-Romo MA, Escalante-Pulido JM, Martínez-Zúñiga R and Ramírez-Arriola MC (2003). Osteoporosis en mexicanas mayores de 40 años. Determinación por densitometría periférica. Rev. Med. IMSS 41: 193-202.

Miller SA, Dykes DD and Polesky HF (1988). A simple salting out procedure for extracting DNA from human nucleated cells. Nucleic Acids Res. 16: 1215.

Murrillo-Uribe A, Delezé-Hinojosa M, Aguirre E, Villa A, et al. (1999). Osteoporosis in Mexican postmenopausal women. Magnitude of the problem. Multicenter study. Ginecol. Obstet. Mex. 67: 227-233.

OMIM (2012). Online Mendelian Inheritance in Man. Johns Hopkins University, Baltimore, MD. MIM Number: $\{601769\}$. Available at [http://www.ncbi.nlm.nih.gov/omim/]. Accessed December 10, 2012.

Qin G, Dong Z, Zeng P, Liu M, et al. (2013). Association of vitamin D receptor BsmI gene polymorphism with risk of osteoporosis: a meta-analysis of 41 studies. Mol. Biol. Rep. 40: 497-506.

Rubi-Castellanos R, Martínez-Cortés G, Muñoz-Valle JF, González-Martín A, et al. (2009). Pre-Hispanic Mesoamerican 
demography approximates the present-day ancestry of Mestizos throughout the territory of Mexico. Am. J. Phys. Anthropol. 139: 284-294.

Sen SS, Rives VP, Messina OD, Morales-Torres J, et al. (2005). A risk assessment tool (OsteoRisk) for identifying Latin American women with osteoporosis. J. Gen. Intern. Med. 20: 245-250.

Silva-Zolezzi I, Hidalgo-Miranda A, Estrada-Gil J, Fernandez-Lopez JC, et al. (2009). Analysis of genomic diversity in Mexican Mestizo populations to develop genomic medicine in Mexico. Proc. Natl. Acad. Sci. U. S. A. 106: 86118616.

Tamayo J, Díaz R, Lazcano-Ponce E, Muñoz M, et al. (2009). Reference values for areal bone mineral density among a healthy Mexican population. Salud Publica Mex. 51 (Suppl 1): S56-S83.

Thakkinstian A, D'Este C, Eisman J, Nguyen T, et al. (2004). Meta-analysis of molecular association studies: vitamin D receptor gene polymorphisms and BMD as a case study. J. Bone Miner. Res. 19: 419-428.

Zintzaras E, Rodopoulou P and Koukoulis GN (2006). BsmI, TaqI, ApaI and FokI polymorphisms in the vitamin D receptor (VDR) gene and the risk of osteoporosis: a meta-analysis. Dis. Markers 22: 317-326. 